\title{
Paper
}

\section{BumpMarker: a 3D-printed tangible marker for simul- taneous tagging, tracking, and weight measurement}

\author{
Changyo Han ${ }^{\dagger}$, Takeshi Naemura (member) ${ }^{\dagger}$
}

\begin{abstract}
This paper introduces BumpMarker: a 3D-printed tangible marker that can perform simultaneous tagging, position tracking, and weight measurement of objects on pressure sensor sheets. The markers baseplate features several pins (raised dots) whose locations encode embedded information. A matrix pressure sensor sheet captures the pressure map of a marker-attached object on a sheet. The embedded data and object weight can be retrieved by processing the pressure map. We propose our design to achieve robust detection of the pins. We also show that our system has the ability to monitor weight changes in tagged objects. Through a series of evaluations, we investigate the technical feasibility of BumpMarker.
\end{abstract}

Key words: Digital fabrication, 3D printing, tangible marker, pressure sensing

\section{Introduction}

In modern society, there is a tremendous demand for inventory management, e.g., in warehouses or hospitals. In inventory management systems, it is necessary to check the names and positions of items. Attaching machine-readable barcodes to them reduces the amount of manual labor needed to monitor quantity. However, storage locations must first be determined in order to find the desired items. Instead, we can track items' positions by attaching radio-frequency identification (RFID) tags to them and placing RFID readers so as to cover the whole storage area*.

However, when we manage containers that store relatively small objects (pellets or powders) or liquids, we must introduce an additional procedure to monitor changes in the amounts of objects in the containers. For example, if we remove a certain amount of liquid chemical from a container, we first identify the label of the container by scanning the attached machine-readable tag. After removing the desired amount of liquid from the container, the mass of the subtracted chemical is measured with a weighing scale. The amount consumed is then recorded for management purposes. This procedure is not desirable since it requires substantial manual

Received June 10, 2018; Accepted August 11, 2018

$\dagger$ The University of Tokyo

(Tokyo, Japan)

This paper includes videos. Note that the videos are not viewable from this PDF file. The videos are available as separate files on the website that hosts this PDF file.

* Teijin Recopick, https://www.teijin.co.jp/focus/recopick/, accessed 2018/06/07.
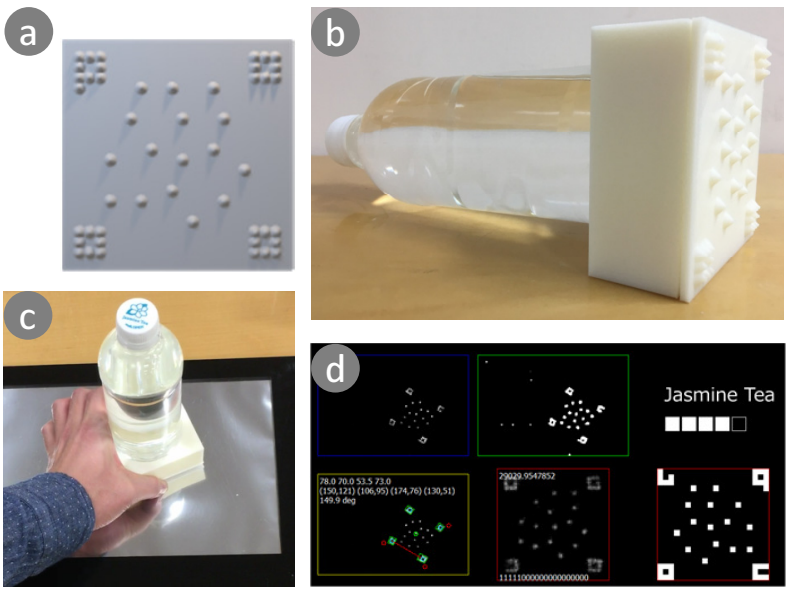

Fig. 1 The proposed pipeline for fabrication and recognition using BumpMarker. (a) A 3D model of a BumpMarker is generated with the embedded data. (b) To tag an object, we attach the printed marker to the bottom of the object. (c) As a user places the object on a pressure sensor sheet, the pressure distribution of the marker's bottom surface is read. (d) The position, the embedded data, and the weight of the object are retrieved by decoding the captured pressure map.

labor and additional devices.

In short, the requirements of inventory management systems include:

- Tags: Machine-readable tags to identify items.

- Position tracking: Track relocated positions of items.

- Weight monitoring: Record the weight changes of containers.

In this paper, we present BumpMarker, a 3D-printed tangible marker enabling simultaneous acquisition of an object's embedded information, position, and weight 
Table 1 Comparison of BumpMarker with other tagging techniques

\begin{tabular}{|l|c|c|c|}
\hline & Barcode & RFID & BumpMarker \\
\hline \hline Machine-readable tag & $\sqrt{ }$ & $\sqrt{ }$ & $\sqrt{ }$ \\
Coarse position tracking & & $\sqrt{ }$ & $\sqrt{ }$ \\
Precise position tracking & & & $\sqrt{ }$ \\
Weight measurement & & & $\sqrt{ }$ \\
\hline
\end{tabular}

on high-resolution pressure sensor sheets ${ }^{1)}$. Figure 1 shows the pipeline for producing and reading a BumpMarker. We adopted 3D printing technique to fabricate BumpMarker because 3D printing has the advantage of rapidly producing prototypes and allowing them to be tested over a short number of iterations. BumpMarker has a simple structure consisting of several pins on a flat baseplate. By modifying the arrangement of the pins, we can embed digital information that can be used as the object's ID. The printed marker is then attached to the bottom of an object. As the object is placed on a pressure sensor sheet, the sheet captures the pressure map and then processes it to track the marker's position, retrieve the information, and calculate the weight of the object. Thus, BumpMarker enables us to remotely monitor the amounts of products in containers by simply placing their containers on the pressure sensor sheet without requiring an additional procedure.

\section{Related Work}

\subsection{Limitations of Barcodes \& RFIDs}

Table 1 shows the comparison of other conventional tagging techniques to our proposed BumpMarker.

Barcodes have been used in logistics and inventory management systems since the 1960s because they are very easy to implement and read. Barcodes consist of several parallel lines and represent data by varying the widths and spacings of the lines. They can be printed easily using printers and can be attached onto an object's surface. Barcodes are usually read by barcode scanners positioned in close proximity, which makes it difficult to track the positions of tagged objects. Additionally, an objects weight must be managed using an additional procedure, as mentioned in the previous section.

RFIDs are typically used as an alternative to barcodes for tagging objects. An RFID tag establishes communication with RFID readers by collecting electromagnetic energy from the readers. We can also obtain the coarse positions of recognized tags if we cover the whole storage area with RFID antennas. However, we still cannot monitor an object's weight while recog- nizing it.

Our proposed BumpMarker system is based on deployed pressure sensor sheets. Since we utilize pressure maps to identify objects, we can accomplish recognition, precise position tracking, and weight measurement simultaneously.

\section{2 Detecting objects on interactive surfaces}

There have been many studies investigating how to detect objects on interactive surfaces such as diffuseillumination multi-touch tables or capacitive touchscreens.

First, we discuss a diffuse-illumination multi-touch table employing infrared (IR) light sources and an IR camera underneath the table. This system can detect touch inputs on the tabletop by capturing an image of the reflected IR light of the diffuse table surface ${ }^{2)}$ ). Thus, markers with distinctive bottom patterns can be distinguished by processing the obtained image. The ability to sense object deformation ${ }^{5)}$ or rearrange the images on surface ${ }^{6)}$ can be achieved by using a bundle of optical fibers. Another study showed the feasibility of detecting the pressure applied to tangible objects using a silicone hemisphere on the marker surface ${ }^{3)}$. However, diffuse-illumination multi-touch tables occupy a substantial amount of space beneath the table, so they are not suitable for inventory management systems.

Second, we consider combining objects with conductive materials, which enables us to detect an object on a capacitive touchscreen ${ }^{7}{ }^{14)}$. The spatial arrangement of the detected touch points is usually used to embed information about the object. However, to persistently detect the touch points, the conductive material touching the touchscreen must be connected to a human body or a ground plane. To detect tangible objects on capacitive touchscreens without human touch, approaches using active electrical circuits $^{8) 10)}$, passive conductive bridges $^{9)}$, or near-field communication (NFC) stickers ${ }^{15)}$ have been proposed. Capacitive touchscreens still have some limitations. Since they utilize capacitance to find touch points, an appropriate distance is required to discriminate between touch points: $1.0 \mathrm{~cm}$ in the case of the $3 \mathrm{M}$ display $^{10)}$. This restricts the size of the marker to a certain amount of embedded information.

\section{3 Matrix pressure sensor sheets}

Matrix pressure sensor sheets are another option for sensing touch on surfaces. The size of a sheet can range from palm-sized objects ${ }^{16)}$ to human-scale objects ${ }^{17)}$. Owing to recent developments in printed electronics technologies, fabricating flexible pressure sensors at low 


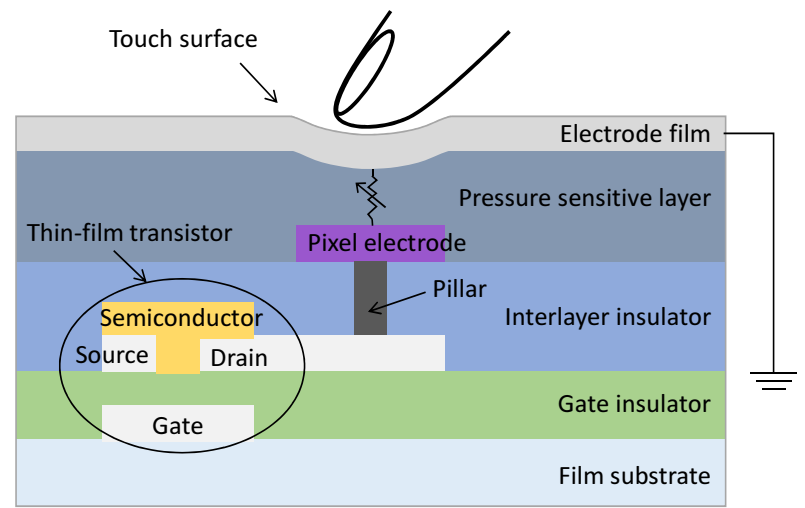

Fig. 2 Cross-sectional view of a printed pressuresensitive sensing element.

Table 2 Specifications of the pressure sensor sheet.

\begin{tabular}{|l|r|}
\hline Item & Value \\
\hline \hline Active area & $173 \mathrm{~mm} \times 260 \mathrm{~mm}$ \\
Number of sensors & $144 \times 216$ \\
Pixel pitch & $1.2 \mathrm{~mm}$ \\
Bit resolution & $8 \mathrm{bit}(0-255)$ \\
Pressure range & $0-250 \mathrm{kPa}$ \\
\hline
\end{tabular}

$\operatorname{cost}^{18)}$ and with high resolution ${ }^{1)}$ is anticipated. Such sheets are expected to be commercialized in the near future, and there is already a commercially available pressure sensor sheet for musical applications * These sensors have great advantages in sensing objects that contains no conductive materials. Additionally, they can distinguish between two touch points at high resolution compared to capacitive touchscreens since they usually monitor the resistance of each sensor pixel. Furthermore, it is expected that they can determine the weight of an object on a sheet sensor with proper postprocessing. To the best of our knowledge, there have been no attempts to design markers that are recognized on matrix pressure sensor sheets.

\section{Pressure sensor sheet}

In this section, we introduce the specifications of the pressure sensor sheet and the design problems encountered in designing the proposed BumpMarker system.

\section{1 Sheet specifications}

We designed the specific parameters of the marker considering the specifications of the pressure sensor sheet, which is supplied from the Japan Advanced Printed Electronics Technology Research Association (JAPERA), as described below. The supplied sheet is fabricated with printed electronics technology as described in the published work ${ }^{1}$.

The structure of a pressure-sensitive sheet sensor is illustrated schematically in Fig. 2. When pressure

\footnotetext{
* Sensel Morph, https://sensel.com/, accessed 2018/06/06.
}

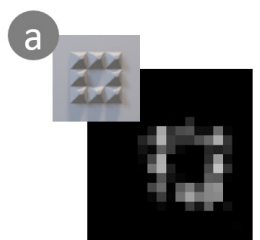

Blurry image

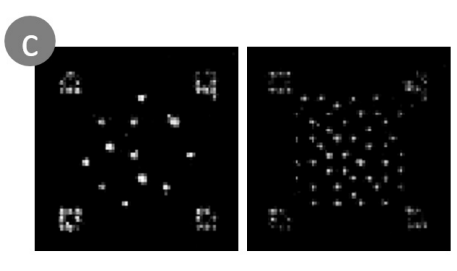

Pin intensity

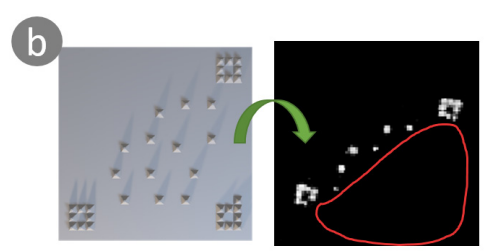

Undetected pins
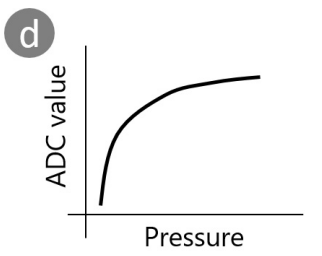

Sensor nonlinearity
Fig. 3 Design problems: (a) blurry images, (b) shifts in the center of mass, (c) intensity changes with weight, and (d) pressure sensor nonlinearity.

is applied to the touch surface, the contact resistance between the touch surface and the pixel electrodes decreases in proportion to the pressure. The change in resistance is sensed by a thin-film transistor (TFT) array on the layer below. Each sensing pixel can measure applied pressures up to $250 \mathrm{kPa}$ with an 8-bit resolution. The sheet sensor has the ability to sense fine surface patterns. It contains over 30,000 sensing elements in a $2 \mathrm{D}$ array and the pitch between the pixels was $1.2 \mathrm{~mm}$ (Table 2).

\section{2 Design problems}

A pressure sensor sheet captures the pressure map of the active area of the sheet as a grayscale image. Unlike images taken by cameras, pressure map images have several distinctive characteristics which should be considered in design. We introduced four problems to consider how the designed markers work on pressure sensor sheets.

\section{(1) Problem 1}

First, unlike visible markers such as QR codes, whose images consists of relatively clean black and white pixels, the captured pressure map is often blurry. This is due to deformation of the pressure-sensitive layer, which causes the applied pressure to distribute to adjacent pixels. It is hard to separate the pins from the obtained image, as shown in Fig. 3 (a).

(2) Problem 2

Second, a biased arrangement of pins shifts the centroid of the marker. This is not a problem in visionbased 2D markers, but in our system, this results in undetected pins, as shown in Fig. 3 (b), which cause bit errors. 


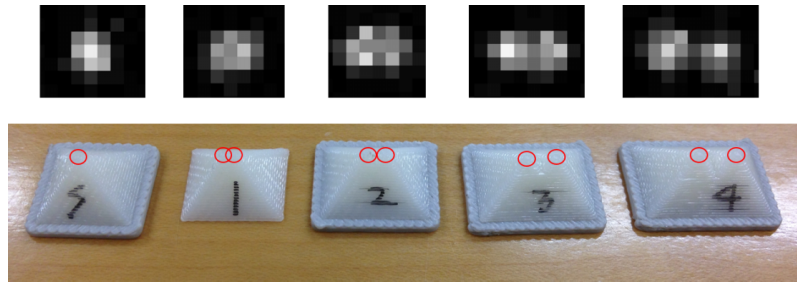

Fig. 4 Captured pressure maps with different pin distances. From left to right, a single pin and two pins separated by one, two, three, and four pixels.

\section{(3) Problem 3}

Third, the intensity of the captured pressure map is affected by the weight of the object. At a certain weight, if we increase the number of pins to embed more data in the marker, the intensity for each pin decreases, which results in sensitivity degradation (Fig. 3 (c)). Moreover, for a marker with certain number of pins, the sensitivity is judged by the load weight.

\section{(4) Problem 4}

Finally, the pressure sensors on the sheet have nonlinear characteristics with respect to the applied force (Fig. $3(\mathrm{~d})$ ). For accurate weight measurement, we should compensate for such nonlinearities.

\section{Design proposal}

In this section, we introduce four design proposals, each aiming to solve one of the design problems mentioned in the previous section.

\section{1 Marker design \& image processing}

We first aimed to investigate the appropriate distance for distinguishing two neighboring pins in order to determine the grid size needed to cope with Problem 1. We modeled conically shaped pins; thus, the pins sat stably on the baseplate. The top of the pins had a circular shape with a diameter of $0.8 \mathrm{~mm}$; this was designed in order to fit the pins into the pixel interval of $1.2 \mathrm{~mm}$. The diameter was also determined by the nozzle diameter $(0.4 \mathrm{~mm})$ of the $3 \mathrm{D}$ printer. We then modeled test samples with two neighboring pins with different distances. After printing, we placed the samples on the sheet sensor and applied a force of approximately $1 \mathrm{~N}$ (0.1 kgf). Although we designed the size of the tip to be smaller than the pixel pitch, the obtained pressure map shows that the pressure spread to surrounding pixels (Fig. 4). We determined the grid size as the distance between three pixels $(3.6 \mathrm{~mm})$ and placed the pin on the center of a grid cell considering that the two pins are visually distinguishable, as shown in Fig. 4.

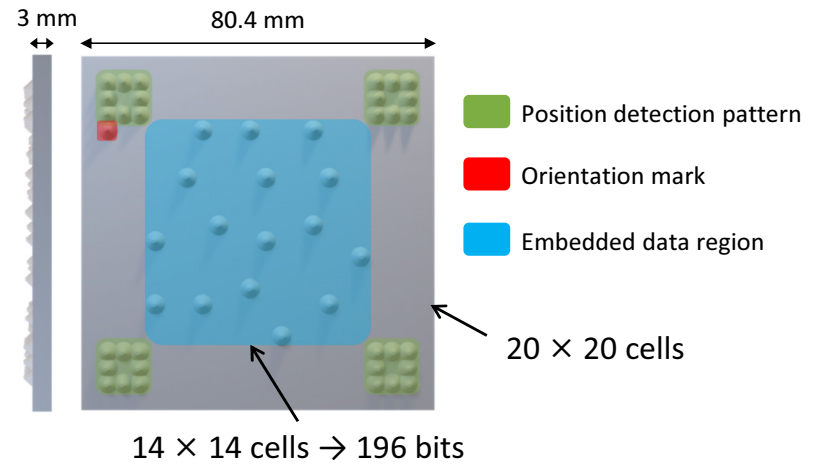

Fig. 5 Structure of BumpMarker with several pins on a square baseplate. The green, red, and blue areas, respectively, show the positions of detected patterns, orientation marks, and embedded data bits.

As the next step, we determined the size of the marker. We utilized polyethylene terephthalate (PET) bottles as containers as a proof of concept. We set up a grid of $20 \times 20$ pins arranged at $3.6-\mathrm{mm}$ intervals to correspond to the typical sizes of the PET bottles. This resulted in the baseplate shape of a square with a side length of $80.4 \mathrm{~mm}$, as shown in Fig. 5. The physical size of the markers could be altered to accommodate more data bits.

We then designed a QR-code-inspired position detection pattern used to find a marker's position on the sheet. A position detection pattern was designed to have eight pins surrounding an empty cell. Unlike QR codes, whose position detection patterns are placed at only three corners, the patterns are situated at four corners of the baseplate in the red areas, as shown in Fig. 5. This was done to maintain an even pressure distribution over the entire marker area because unevenly distributed pins would cause the pressure to be biased at certain marker positions, as mentioned in Problem 2. This brings a point symmetry to the marker that makes the orientation indistinguishable. Hence, we added a single pin next to the position detection pattern to designate the orientation of the marker. The blue area was used to contain up to 196 bits of embedded data. We placed a pin to represent a '1' bit and an empty space to represent a '0' bit.

The decoding process of the BumpMarker system is shown in Fig. 6. The pressure map of the entire sheet area was continuously captured as a grayscale image of $144 \times 216$ pixels at $100-\mathrm{Hz}$ intervals.

In the first stage, we processed the raw image to make it clearer for the next stage. We took the first $100 \mathrm{im}$ age frames and averaged them to obtain the initial pixel 


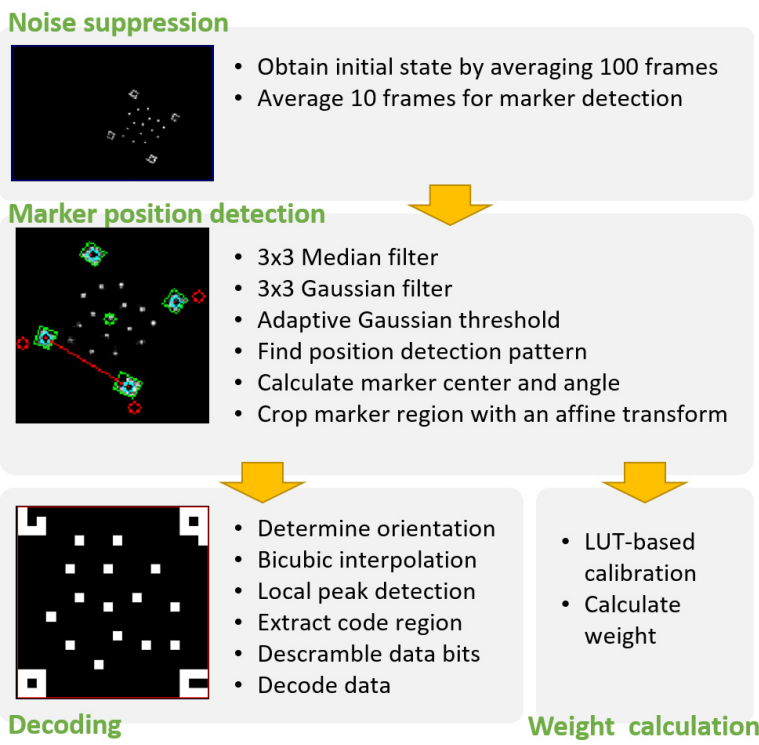

Fig. 6 The reading and decoding process of the proposed BumpMarker system

values of the sheet sensor and removed background electrical noise. The obtained initial state was used for calibration afterwards by subtracting it from the measured frames. Next, we averaged and normalized ten recent frames and adopted them for marker detection.

Next, we detected the marker locations using the rectified image from the previous stage. After the filtering process described in Fig. 6, the image was binarized by adaptive Gaussian thresholding in order to cope with the varying intensity of the image. After extracting contours, the decoder searched for nested contours and classified them as position detection patterns. Since a position detection pattern is susceptible to damage and often loses its nested pattern, we regarded blob contours over a certain area as position detection patterns to increase the robustness of detecting the marker region. Using the detected patterns, we calculated the marker's center coordinates and rotation. We then eliminated the marker's rotation and cropped the marker region of $69 \times 69$ pixels from the image from the previous stage by an affine transform.

With the cropped marker image, we first determined the orientation of the marker and rotated it to the desired rotation. The cropped image was used to calculate the weight afterwards. After resampling the image five times using bicubic interpolation ${ }^{7)}$, local peak detection was performed for precise estimation of the grid and pins. The threshold for local peak detection was 30 in every case. The grid center was then estimated by folding the entire marker region by 15 pixels. Af-

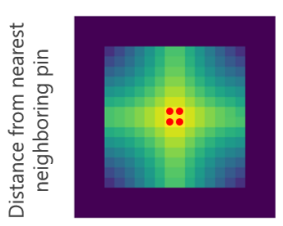

Step 1

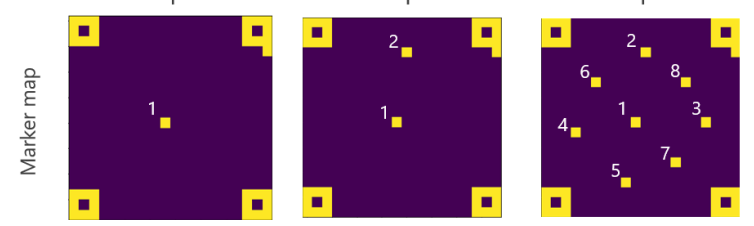

Fig. 7 Orders and locations of the data bits scrambled by the code scrambler. Each picture shows the order of data bits in the corresponding step. ter shifting the image to the grid center, we achieved 196 bits of embedded data by descrambling the $14 \times$ 14 pixel binary image.

\section{2 Code scrambler}

We propose a code scrambler to manage the shift in the centroid (Problem 2). Let us assume that the applied pressure on the baseplate is evenly distributed over the marker area. In a specific area of the marker, if there is larger number of pins, less pressure is distributed over each pin. Therefore, we cannot naively place the pins at the edges as in QR codes because this would lead to undetected pins. To make the pins more effective at lower object weights, the pins should be scattered over the data region. Furthermore, as mentioned in the design problems section, the data pins should be distributed as evenly as possible to prevent biasing the pressure distribution.

However, it is difficult to evenly distribute the pins for an unknown data bit length. It is even more difficult to do so where the data bit sequence has 0 and 1 values in a random order.

To solve the above-mentioned problems, we designed a code scrambler that evenly scatters data bits over the $2 \mathrm{D}$ plane of the marker's surface. We assumed that all data bits had values of '1' to simplify the conditions. The code scrambler determines the order of data bits in the following manner:

(1) For each step, locate a pin (bit) for a grid cell where the distance from the nearest neighboring pin is a maximum.

(2) If there multiple candidates satisfy condition (1), choose a grid cell that makes the centroid as close as possible to the center of the marker.

The operation of the scrambler is depicted in Fig. 7 . The upper figures show distance from the nearest ad- 
jacent pin for each step, and the lower figures show the arrangement of the pins after the step. The numbers annotated on the pins represent the order of the input bit sequence before scrambling. For example, let us suppose that we embed 8-bit data. In step 1 (the upper left picture), there are four position candidates, marked as red dots, satisfying condition (1) of the scrambler's policy. The lower left position is then chosen because it makes the center of mass closest to the center of the marker. In step 2 (the upper middle picture), there is only one position candidate satisfying condition (1). Repeating the code scrambling algorithm, the 8-bit data is distributed in the order represented in the lower right panel of Fig. 7. The contribution of the scrambler to enhancing the pin detection rate is discussed later in the evaluation section.

\section{3 Available number of pins versus weight}

We propose a method of dealing with Problem 3. As mentioned in the previous section, the intensity of the captured pressure map depends on object weight. Thus, for the same number of pins with a different object weight, the intensity of each pin changes, which affects pin detection sensitivity. Similarly, for the same weight with a different number of pins, the pressure applied to each pin changes. Since it is difficult to simulate every condition of contact between the marker and the pressure sensor, we experimentally investigated the effect of the number of pins and object weight on pin detection sensitivity.

\section{4 Nonlinearity compensation for weight calculation}

Suppose that the total force acting between the sheet sensor and the object is only the gravitational force; then the weight of the object can be estimated by fitting the sum of the pixel values to the actual weight. There is a nonlinear relationship between the resistance of the pressure-sensitive layer and the applied pressure (Problem 4). Therefore, we experimentally investigated the representative characteristics of a single pressuresensing pixel by applying pressure over a fixed area. We then built a lookup table (LUT) to linearize the obtained pixel values by fitting the obtained characteristics with a fourth-order polynomial. After calibrating the pixel values, their sum in the marker region was calculated. Finally, the sum was linearly fitted to the actual weight by applying a constant gain.

\section{Evaluation}

In this section, we present the experimental results

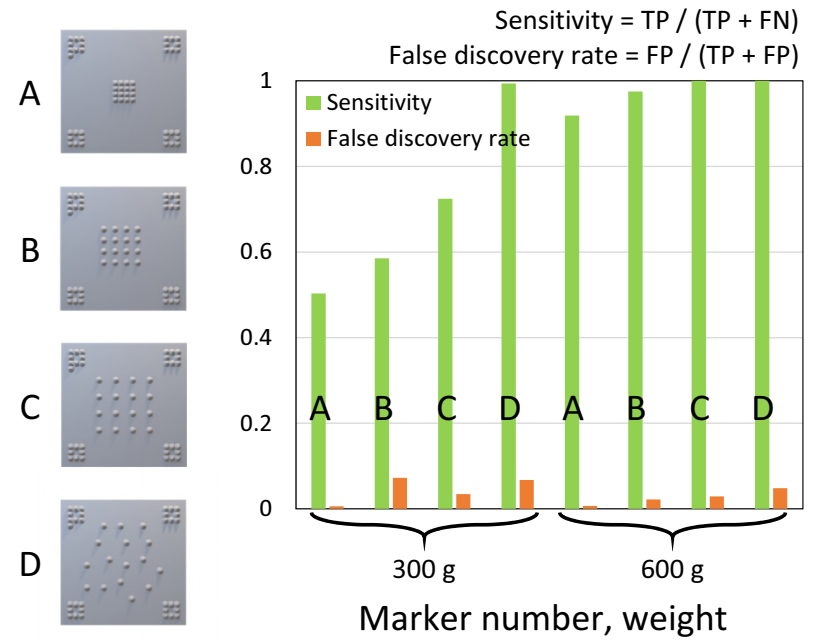

Fig. 8 Pin detection sensitivity with different pin arrangements and the same number of pins.

to validate our BumpMarker system. We set up the pressure-sensitive sheet sensor on a flat, level table and put markers on the sensor to perform measurements.

We implemented the whole design process into an Autodesk Fusion $360^{*}$ script. The script took user-specific data bits as input and automatically generated a 3D model of the BumpMarker system (Fig. 1 (a)). We also designed an adapter that uniformly distributed the weight of the bottle over the marker (Fig. 1 (b)).

We fabricated BumpMarkers and adapters with Stratasys Dimension 1200es, a fusion deposition modeling (FDM) 3D printer with a 0.254-mm layer thickness. The XY plane accuracy was not specified, but we observed that the printer was accurate enough to print separate pins at a $3.6-\mathrm{mm}$ pitch. We used a rigid ABS material to print the designed marker.

\section{1 Marker recognition}

We implemented the decoding algorithm described in section 4.1 with the graphical user interface shown in Fig. $1(\mathrm{~d})$. The upper left picture shows the raw grayscale image of the captured pressure map. After detecting the position of the marker as shown in the lower left picture, we extracted the marker region as shown in the lower middle picture. Finally, after local peak detection, we obtained a $20 \times 20$ pixel binary image as illustrated in the lower right section which appeared clear enough to be decoded.

\subsection{Pin detection sensitivity with different pin arrangements}

In order to validate the effect of the scrambler, we fabricated four kinds of testing samples as illustrated in the left of Fig. 8. All markers had 16 pins in the

\footnotetext{
* https://www.autodesk.com/products/fusion-360/overview
} 


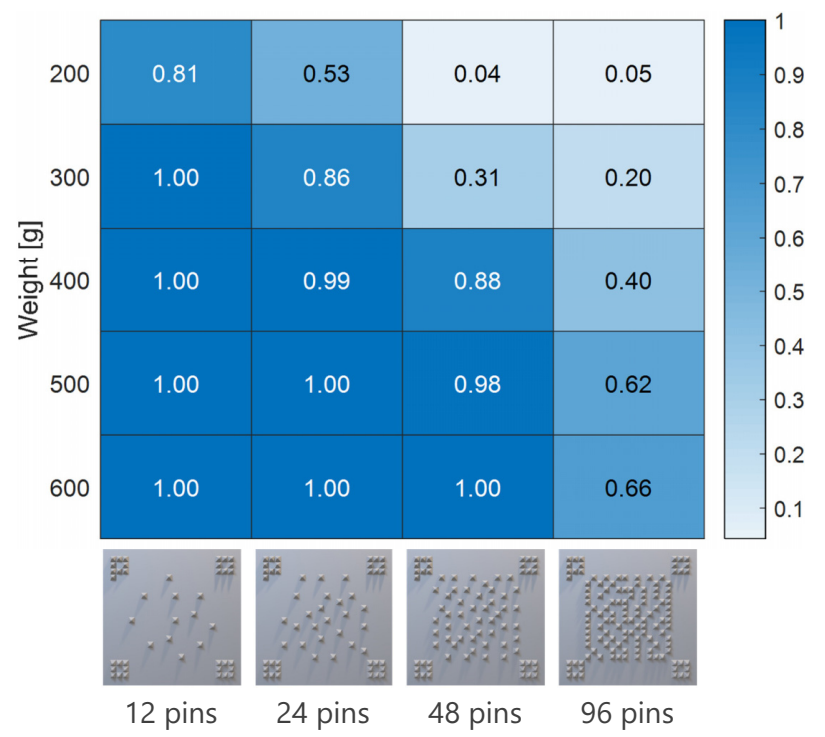

Fig. 9 Pin detection sensitivity with various numbers of pins and object weights.

code region, but the arrangement of the pins was different. In marker $\mathrm{A}$, the pins were placed densely in the center of the marker with the distance of $3.6 \mathrm{~mm}$ from the neighboring pins. For marker B, the distance was $7.2 \mathrm{~mm}$, but the pins were slightly off-center because the data region had $14 \times 14$ points. Marker $\mathrm{C}$ had evenly distributed pins with a $10.8-\mathrm{mm}$ pitch. For marker D, the pins were arranged by the scrambler as mentioned in the marker design section. To observe the transition of the detection rate depending on object weight, we tested two object masses: $300 \mathrm{~g}$ and $600 \mathrm{~g}$.

In the measurement, we put the marker at a random location on the sheet sensor and investigated the number of true positives and false positives. The measurements were taken 20 times for each weight and pin arrangement. The result is illustrated in the right of Fig. 8. The sensitivity represents the sum of true positives divided by sum of true positives and false negatives. The sensitivity increased as the distance between the pins increased. Marker D showed a similar detection rate compared to marker $\mathrm{C}$ when the weight was $600 \mathrm{~g}$ and showed the best performance among all the markers at $300 \mathrm{~g}$. In all conditions, false discovery rates were less than $10 \%$. These false negatives arose from the local peak detector because it recognized small amounts of noise as pins.

\section{3 Available number of pins versus weight}

We printed four markers with different numbers of pins to test samples as shown at the bottom of Fig. 9. For each marker, we measured the detection sensitivity of the pins with masses of $200 \mathrm{~g}$ to $600 \mathrm{~g}$ in 100-g incre-

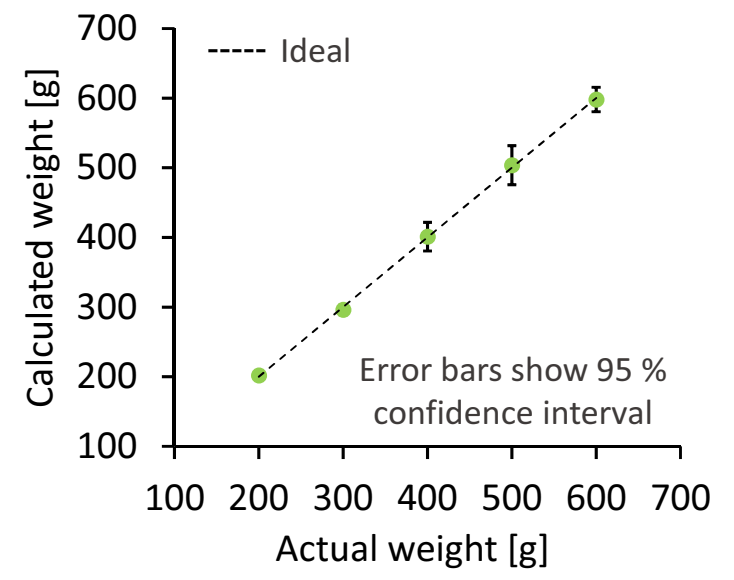

Fig. 10 Calculated weight as a function of actual weight.

ments. The results are illustrated at the top of Fig. 9. The pin detection sensitivity decreased with decreasing weight. On the other hand, the sensitivity decreased as the number of pins increased. Designing a marker aiming for a certain sensitivity, we could determine the available number of pins (bit length) by reading the results. The result is applicable for the specific sheet used in this case, but comparable results could be derived by conducting similar measurements using other sheets.

\section{4 Weight measurement accuracy}

Considering only the gravitational force, the pressure applied to the sheet sensor showed a linear relationship between with the object weight. However, it was difficult to establish a physical model because there were too many factors to be considered. For example, if a marker pin were placed between the sensing elements of the sensor, the measured pressure values would differ compared with a case in which a pin were placed directly on the top of the sensor electrode.

Thus, we experimentally derived the relationship between the actual weight and the measured weight. We measured the sum of the compensated pixel values in the marker region with weights of $200 \mathrm{~g}$ to $600 \mathrm{~g}$ at $100-\mathrm{g}$ increments. We excluded the 100-g-weight case because the captured image was not clear enough, leading to failure to find the position of a marker. Each measurement was done 16 times by placing the marker $\mathrm{D}$ in Fig. 9 on the random position of the sheet. The measured data points for each weight were averaged and we linearly fitted the points using the actual weight of the marker.

Figure 10 shows the calculated weight as a function of actual weight. The green dots imply the averaged weight of the marker and the error bars represent $95 \%$ 
confidence intervals for the corresponding weight. The errors were relatively larger for heavier loads. This indicated that saturated pixel values might lead to weight errors. However, the results show that our BumpMarker system is capable of distinguishing weight differences of $100 \mathrm{~g}$ in most cases.

\section{Application scenarios}

We introduce expected application scenarios in this section.

The BumpMarker system can be set up easily by laying out the thin-pressure-sensor sheet on a flat surface such as a table or a shelf. For example, if storing various liquid medicines on shelves in a hospital, the proposed system can automatically monitor the precise positions of the medicines and their remaining amounts. This is expected to greatly reduce the effort required to manage such items.

Supposing that the cost of the pressure sensor sheet decreases, we can implement the proposed system in refrigerator shelves to check which food or beverage supplies are running out. Moreover, by combining the data with electronic commerce, the proposed system could automatically purchase items that are out of stock.

The BumpMarker system can be produced not only with a $3 \mathrm{D}$ printer but with conventional mass production processes such as injection molding or blow molding. If there is a standard for physical markers, products' IDs can be embedded in the bottoms of containers throughout the production process. With a BumpMarker-powered inventory management system, users can manage their stock with reduced effort.

\section{Discussion and future work}

We focused on embedding variable lengths of information in this study, but with a fixed number of pins, it is possible to embed considerably different kinds of IDs by combining pin arrangements. As a visible augmented reality (AR) marker, Uchiyama et al. suggested extracting key points from random dot patterns to distinguish markers ${ }^{19)}$. This approach is not directly applicable to the proposed marker because we must consider the pressure distribution problem mentioned above. We plan to further investigate possible options to apply such methods with our marker design.

As mentioned in the marker design section, we already use 33 pins to represent the positions of the markers. This is a considerable amount, as we could use only 12 pins for data representation at a weight of $300 \mathrm{~g}$, as shown in Fig. 9. An alternative proposal for estimating marker positions and rotations is needed in order to enhance the information-embedding feasibility of the proposed marker.

\section{Conclusion}

We have introduced a 3D-printed tangible marker called BumpMarker that enables simultaneous tagging, position tracking, and weight measurement of an object. We contributed to establishing a design method for a physical marker that can operate on a pressure sensor sheet. We confirmed experimentally that we could enhance the detection sensitivity of the pins used by scattering them appropriately in space. We also shown that our system can distinguish approximately $100 \mathrm{~g}$ of weight difference. With our proposed BumpMarker system, we expect to implement inventory management systems that can monitor the locations and weights of items remotely.

\section{Acknowledgement}

This work was partially supported by the New Energy and Industrial Technology Development Organization, Japan (NEDO).

\section{References}

1) Hitoshi Kondoh, Toru Miyoshi, Shinichi Nishi, and Toshihide Kamata : "Multifunctional Flexible Sheet Sensor Using Printing Technologies", In IDW '17 pp. 1487-1490 (2017)

2) Sergi Jordà, Gnter Geiger, Marcos Alonso, and Martin Kaltenbrunner : "The reacTable: exploring the synergy between live music performance and tabletop tangible interfaces", In Proceedings of the 1st international conference on Tangible and embedded interaction - TEI '07 pp. 139-146 (2007)

3) Fabian Hennecke, Franz Berwein, and Andreas Butz : "Optical pressure sensing for tangible user interfaces", In Proceedings of the ACM International Conference on Interactive Tabletops and Surfaces - ITS '11 pp. 45-48 (2011)

4) Cary Williams, Xing Dong Yang, Grant Partridge, Joshua MillarUsiskin, Arkady Major, and Pourang Irani : "TZee: Exploiting the Lighting Properties of Multi-touch Tabletops for Tangible 3D Interactions", In Proceedings of the 2011 annual conference on Human factors in computing systems - CHI '11 pp. 1363-1372 (2011)

5) Kentaro Go, Katsutoshi Nonaka, Koji Mitsuke, and Masayuki Morisawa : "Object shape and touch sensing on interactive tables with optical fiber sensors", In Proceedings of the Sixth International Conference on Tangible, Embedded and Embodied Interaction - TEI '12 pp. 123-126 (2012)

6) Patrick Baudisch, Torsten Becker, and Frederik Rudeck : "Lumino: tangible blocks for tabletop computers based on glass fiber bundles", In Proceedings of the 28th international conference on Human factors in computing systems - CHI '10 pp. 1165-1174 (2010)

7) Jun Rekimoto and Jun : "SmartSkin: an infrastructure for freehand manipulation on interactive surfaces", In Proceedings of the SIGCHI conference on Human factors in computing systems Changing our world, changing ourselves - CHI '02 pp. 113-120 (2002)

8) Simon Voelker, Christian Cherek, Jan Thar, Thorsten Karrer, Christian Thoresen, Kjell Ivar Øvergård, and Jan Borchers : "PERCs: Persistently Trackable Tangibles on Capacitive MultiTouch Displays", In Proceedings of the 28th Annual ACM Sym- 
posium on User Interface Software \& Technology - UIST '15 pp. 351-356 (2015)

9) Simon Voelker, Kosuke Nakajima, Christian Thoresen, Yuichi Itoh, Kjell Ivar Øvergård, and Jan Borchers : "PUCs: Detecting Transparent, Passive Untouched Capacitive Widgets on Unmodified Multi-touch Displays", In Proceedings of the 2013 ACM international conference on Interactive tabletops and surfaces ITS '13 pp. 101-104 (2013)

10) Neng-Hao Yu, Polly Huang, Yi-Ping Hung, Li-Wei Chan, Seng Yong Lau, Sung-Sheng Tsai, I-Chun Hsiao, Dian-Je Tsai, Fang-I Hsiao, Lung-Pan Cheng, and Mike Chen : "TUIC: Enabling Tangible Interaction on Capacitive Multi-touch Display", In Proceedings of the 2011 annual conference on Human factors in computing systems - CHI '11 pp. 2995-3004 (2011)

11) Liwei Chan, Stefanie Müller, Anne Roudaut, and Patrick Baudisch : "CapStones and ZebraWidgets: Sensing Stacks of Building Blocks, Dials and Sliders on Capacitive Touch Screens", In Proceedings of the 2012 ACM annual conference on Human Factors in Computing Systems - CHI '12 pp. 2189-2192 (2012)

12) Kohei Ikeda and Koji Tsukada: "CapacitiveMarker: Novel interaction method using visual marker integrated with conductive pattern", In Proceedings of the 6th Augmented Human International Conference on - AH '15 pp. 225-226 (2015)

13) Martin Schmitz, Jrgen Steimle, Jochen Huber, Niloofar Dezfuli, and Max Mühlhäuser : "Flexibles: Deformation-Aware 3DPrinted Tangibles for Capacitive Touchscreens", In Proceedings of the 2017 CHI Conference on Human Factors in Computing Systems - CHI '17 pp. 1001-1014 (2017)

14) Martin Schmitz, Mohammadreza Khalilbeigi, Matthias Balwierz, Roman Lissermann, Max Mühlhäuser, and Jrgen Steimle : "Capricate: A Fabrication Pipeline to Design and 3D Print Capacitive Touch Sensors for Interactive Objects", In Proceedings of the 28th Annual ACM Symposium on User Interface Software \& Technology - UIST '15 pp. 253-258 (2015)

15) Nicolas Villar, Haiyan Zhang, Daniel Cletheroe, Greg Saul, Christian Holz, Tim Regan, Oscar Salandin, Misha Sra, HuiShyong Yeo Yeo, and William Field : "Project Zanzibar: A Portable and Flexible Tangible Interaction Platform", In Proceedings of the 2018 CHI Conference on Human Factors in Computing Systems - CHI '18 pp. 1-13 (2018)

16) Su-Jeong Woo, Jeong-Ho Kong, Dae-Gon Kim, and Jong-Man Kim : "A thin all-elastomeric capacitive pressure sensor array based on micro-contact printed elastic conductors", J. Mater. Chem. C 2(22):4415-4422 (2014)

17) A. K.M. Mahfuzul Islam, Masamune Hamamatsu, Tomoyuki Yokota, Sunghoon Lee, Wakako Yukita, Makoto Takamiya, Takao Someya, and Takayasu Sakurai : "Programmable neuron array based on a 2-transistor multiplier using organic floating-gate for intelligent sensors", IEEE Journal on Emerging and Selected Topics in Circuits and Systems 7(1):81-91 (2017)

18) Takahiro Hashizume, Takuya Sasatani, Koya Narumi, Yoshiaki Narusue, Yoshihiro Kawahara, and Tohru Asami : "Passive and contactless epidermal pressure sensor printed with silver nanoparticle ink", Proceedings of the 2016 ACM International Joint Conference on Pervasive and Ubiquitous Computing - UbiComp '16 pp. 190-195 (2016)

19) Hideaki Uchiyama and Hideo Saito : "Random dot markers", In Proceedings of the 2011 IEEE Virtual Reality Conference pp. 35-38 (2011)

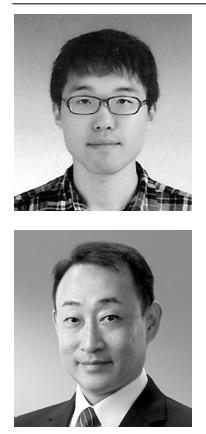

Changyo Han is a Ph. D. candidate at the Information and Communication Engineering Department, the University of Tokyo. His research interests include tangible user interfaces and digital fabrication. He can be contacted at hanc@naelab.org.

Takeshi Naemura is a professor at Interfaculty Initiative in Information Studies, The University of Tokyo. His research interests include Virtual Reality and Human Interface. He is a member of the ITE. He received Niwa-Takayanagi Best Paper Award from ITE, Young Researcher Award from MEXT, Innovative Technologies from METI and so on. He can be contacted at naemura@naelab.org. 